BMJ Open

Diabetes

Research

\& Care

\title{
Elevation of blood glucose level predicts worse outcomes in hospitalized patients with COVID-19: a retrospective cohort study
}

\author{
Jianfeng Wu, ${ }^{1}$ Jianqiang Huang, ${ }^{2}$ Guochao Zhu, ${ }^{3}$ Qiongya Wang, ${ }^{4}$ Qingquan Lv, ${ }^{5}$ \\ Ying Huang, ${ }^{6}$ Yang $\mathrm{Yu},{ }^{7}$ Xiang $\mathrm{Si},{ }^{1} \mathrm{Hui} \mathrm{Yi}^{2}{ }^{2}$ Cuiping Wang, ${ }^{1}$ Yihao Liu (D) , 8,9 \\ Han Xiao, ${ }^{10}$ Qian Zhou, ${ }^{8}$ Xin Liu, ${ }^{11}$ Daya Yang, ${ }^{12}$ Xiangdong Guan, ${ }^{1}$ Yanbing Li (D) , ${ }^{9}$ \\ Sui Peng, ${ }^{8}$ Joseph Sung, ${ }^{13}$ Haipeng Xiao (i) ${ }^{9,14}$
}

To cite: Wu J, Huang J, Zhu G, et al. Elevation of blood glucose level predicts worse outcomes in hospitalized patients with COVID-19: a retrospective cohort study. BMJ Open Diab Res Care 2020;8:e001476. doi:10.1136/ bmjdrc-2020-001476

- Additional material is published online only. To view please visit the journal online (http://dx.doi.org/10.1136/ bmjdrc-2020-001476).

JW, JH, GZ, QW, QL, YH and SP contributed equally.

$\mathrm{JW}, \mathrm{JH}, \mathrm{GZ}, \mathrm{QW}, \mathrm{QL}$ and $\mathrm{YH}$ are joint first authors.

SP and HaiX are joint senior authors.

Received 17 April 2020

Revised 15 May 2020

Accepted 24 May 2020

Check for updates

(c) Author(s) (or their employer(s)) 2020. Re-use permitted under CC BY-NC. No commercial re-use. See rights and permissions. Published by BMJ.

For numbered affiliations see end of article.

Correspondence to Professor Haipeng Xiao; xiaohp@mail.sysu.edu.cn

\section{ABSTRACT}

Introduction With intense deficiency of medical resources during COVID-19 pandemic, risk stratification is of strategic importance. Blood glucose level is an important risk factor for the prognosis of infection and critically ill patients. We aimed to investigate the prognostic value of blood glucose level in patients with COVID-19.

Research design and methods We collected clinical and survival information of 2041 consecutive hospitalized patients with COVID-19 from two medical centers in Wuhan. Patients without available blood glucose level were excluded. We performed multivariable Cox regression to calculate HRs of blood glucose-associated indexes for the risk of progression to critical cases/mortality among noncritical cases, as well as in-hospital mortality in critical cases. Sensitivity analysis were conducted in patient without diabetes.

Results Elevation of admission blood glucose level was an independent risk factor for progression to critical cases/ death among non-critical cases ( $\mathrm{HR}=1.30,95 \% \mathrm{Cl} 1.03$ to $1.63, p=0.026)$. Elevation of initial blood glucose level of critical diagnosis was an independent risk factor for inhospital mortality in critical cases $(\mathrm{HR}=1.84,95 \% \mathrm{Cl} 1.14$ to $2.98, p=0.013)$. Higher median glucose level during hospital stay or after critical diagnosis ( $\geq 6.1 \mathrm{mmol} / \mathrm{L})$ was independently associated with increased risks of progression to critical cases/death among non-critical cases, as well as in-hospital mortality in critical cases. Above results were consistent in the sensitivity analysis in patients without diabetes.

Conclusions Elevation of blood glucose level predicted worse outcomes in hospitalized patients with COVID-19. Our findings may provide a simple and practical way to risk stratify COVID-19 inpatients for hierarchical management, particularly where medical resources are in severe shortage during the pandemic.

\section{BACKGROUND}

The pandemic of COVID-19 is a global health crisis, ${ }^{1}$ with the number of confirmed cases worldwide reaching over 3 million and counting, ${ }^{2}$ as well as an overall case fatality rate of $2.3 \%-9.9 \%$ and mortality as high as

\section{Significance of this study}

What is already known about this subject?

- Previously published articles found that age, gender, comorbidities, lymphocyte counts, $\mathrm{C}$ reactive protein, procalcitonin, D-Dimer and other cytokines were associated with prognosis of patients with COVID-19. However, the effect of blood glucose level on prognosis was never reported.

What are the new findings?

- Elevation of admission blood glucose level was an independent risk factor for progression to critical cases/death among non-critical cases ( $\mathrm{HR}=1.30$, $95 \% \mathrm{Cl} 1.03$ to $1.63, \mathrm{p}=0.026)$. Elevation of initial blood glucose level of critical diagnosis was an independent risk factor for in-hospital mortality in critical cases $(\mathrm{HR}=1.84,95 \% \mathrm{Cl} 1.14$ to 2.98 , $\mathrm{p}=0.013$ ). Higher median glucose level during hospital stay or after critical diagnosis (median glucose $\geq 6.1 \mathrm{mmol} / \mathrm{L}$ ) was independently associated with increased risks of progression to critical cases/death among non-critical cases, as well as in-hospital mortality in critical cases.

How might these results change the focus of research or clinical practice?

- Our findings may provide a simple and practical way to risk stratify COVID-19 inpatients for hierarchical management, particularly where medical resources are in severe shortage during the pandemic.

$49 \%$ in critical cases. ${ }^{2-4}$ Faced with its rapid spread, medical resources, especially for intensive care units (ICUs), are being heavily taxed to the point of near depletion in many countries. Therefore, one of the pressing challenges in its management is the prompt and accurate prognostication of patients with COVID-19 based on their likelihood to progress into critical or life-threatening conditions. Risk stratification would be of immense 
help for clinicians as well as health administrative authorities worldwide to capitalize their limited resources in their response to the outbreak. ${ }^{5}$

However, there was still no universally feasible laboratory index to predict the prognosis of patients with COVID-19, particularly during such a large-scale infectious disease outbreak setting. Blood glucose level is a routine test that can be carried out in virtually all clinical settings. Among patients with COVID-19, 45.2\% had anomalies in blood glucose levels. ${ }^{3}$ Previous studies showed that stress hyperglycemia was an independent risk factor associated with worse outcomes among critically ill patients. ${ }^{6}$ In addition, hypoglycemia may lead to cardiovascular events, also conferring a harmful effect on prognosis. ${ }^{7}$ We therefore speculated that blood glucose levels may have predictive value on COVID-19 prognosis.

In our current study, we reviewed 2041 patients with COVID-19 from two medical centers in Wuhan City and explored the effects of blood glucose levels on the outcomes of progression to critical cases and in-hospital mortality. We sought to identify an instant and simple approach for risk stratification and hierarchical management of COVID-19 in all clinical settings.

\section{RESEARCH DESIGN AND METHODS \\ Study population}

Consecutive inpatients with COVID-19 from Wuhan Hankou Hospital and No. Six Hospital of Wuhan between 26 December 2019 and 15 March 2020 were collected in this study. The final follow-up date was 19 March 2020. Patients without available blood glucose test results were excluded from our study. The disease severity (critical or non-critical) was evaluated dynamically from the admission to the discharged. Critical patients were those who met any of the following conditions during the whole hospital stay: (1) respiratory failure requiring mechanical ventilation; (2) failures of other organs that required monitoring and treatment in ICU; and (3) shock. $^{8}$ Informed consent was waived.

\section{Data collection and definition}

We collected the patients' clinical data, including demographic information, medical history, laboratory findings, treatment and prognosis. Admission blood glucose level was measured from the first blood sample after admission. The initial blood glucose level of critical diagnosis was defined as the first blood glucose level after being diagnosed as critical case. We used occurrence of hypoglycemia, median blood glucose, glucose coefficient of variation, maximum blood glucose and minimum blood glucose to evaluate in-hospital blood glucose status. The level of median blood glucose during hospital stay or after critical diagnosis was categorized into $<6.1 \mathrm{mmol} / \mathrm{L}$ and $\geq 6.1 \mathrm{mmol} / \mathrm{L} .{ }^{9}$ Hyperglycemia was defined as blood glucose level $\geq 6.1 \mathrm{mmol} / \mathrm{L}$ after admission. ${ }^{10}$ Hypoglycemia was defined as blood glucose level below $4.0 \mathrm{mmol} / \mathrm{L}$ after admission. ${ }^{11}$

\section{Study outcomes}

For non-critical patients at admission, we used a composite outcome to analyze the association between glucoserelevant indexes and the risk of progression to critical cases/death. The composite outcome was reached when any of the following outcomes occurred, which included admission to ICU, mechanical ventilation, hemodynamic compromise or death. The time to the composite outcome was identified as the interval from admission to the first occurrence of the composite outcome during hospital stay. For critical patients, we evaluated the predictive performances of glucose-associated indexes in in-hospital mortality. The time from critical cases to death was defined as the time interval from the date diagnosed as critical cases to the date of death from any cause.

\section{Statistical analysis}

All continuous variables were tested for normality. Data were expressed as mean and SD if normally distributed or as median and IQR otherwise. The categorical variables were presented as frequencies and percentages. Cox proportional hazard regression models were applied to evaluate the association between the multiple blood glucose indexes and prognosis. This multivariable analysis was adjusted for all factors included in the univariable analysis. The HRs and 95\% CIs of Cox regression models were reported. Because patients with diabetes generally had higher glucose levels and poor outcomes than that of patients without diabetes, which may result in bias in evaluating the relationship of glucose levels with the rate of progression to critical cases/death or in-hospital mortality. Therefore, in the present study, sensitivity analysis was conducted to evaluate the association of admission blood glucose level with the risk of critical cases/death among non-critical patient without diabetes at admission. Similar analyses were also performed to evaluate the association of initial blood glucose level of critical diagnosis with in-hospital mortality among critical patients without diabetes. We used Stata/MP V.14.0 and $\mathrm{R}$ software (V.3.6.1) to conduct data analyses, and twosided $p$ values less than 0.05 were considered significant.

\section{RESULTS}

\section{Baseline characteristics}

From 26 December 262019 to 15 March 2020, a total of 2289 cases of hospitalized patients with COVID-19 were consecutively collected from two medical centers in Wuhan, China. After exclusion of cases without available blood glucose data $(n=248)$, a total of 2041 cases of hospitalized patients with COVID-19 were collected. The median glucose determination number was 2 (IQR 1-4) for all patients during the whole hospitalization with a median in-hospital stay of 13.5 days (IQR 7.8-20.9), and the determination number of fasting blood glucose for all patients was 2 (IQR 1-3). Among all cases, 697 patients were critical cases, $30.1 \%$ (210/697) patients were diagnosed as critical cases at admission, 49.6\% (346/697) 


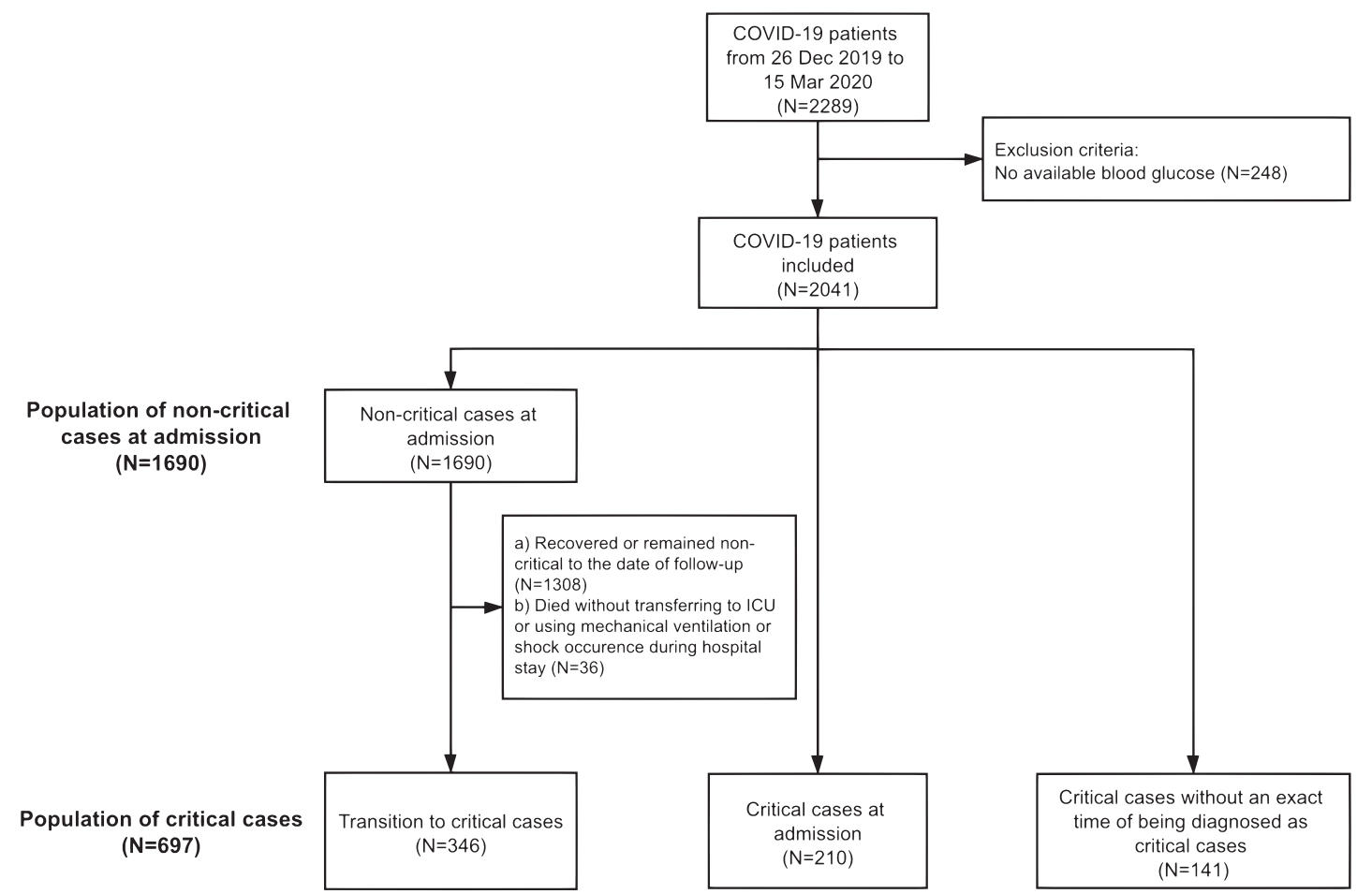

Figure 1 Flow chart of the inclusion and exclusion of patients with COVID-19. ICU, intensive care unit.

patients were developed among non-critical cases at admission and 20.2\% (141/697) patients were diagnosed as critical cases but the exact time of being diagnosed as critical cases was missing. A total of 1690 patients were non-critical at admission. A detailed flow chart was shown in figure 1. Baseline characteristics of all 2041 cases were shown in online supplementary table S1. Table 1 showed the baseline characteristics of non-critical patients at admission and critical cases at the time of critical diagnosis. For non-critical patients at admission, the median age was 61.0 years (IQR 50.0-69.0) and 865 (51.2\%) were female. The median admission blood glucose was $5.9 \mathrm{mmol} / \mathrm{L}$ (IQR 5.1-7.5). Median in-hospital stay was 13.3 days (IQR 7.7-20.9). For critical cases, the median age was 65.0 years (IQR 54.0-73.0), and $322(46.2 \%)$ patients were women. Median initial blood glucose level of being diagnosed as critical cases was $6.9 \mathrm{mmol} / \mathrm{L}$ (IQR 5.7-8.7). Median in-hospital stay was 15.8 days (IQR 8.4-23.1). The 30-day in-hospital mortality was $14.6 \%$ for all patients.

\section{Blood glucose level was an independent risk factor for} progression to critical cases/death among non-critical cases For 1690 non-critical cases at admission, 382 (22.6\%) patients developed into critical cases/death. The 30-day rate of progression to critical cases/death among noncritical cases at admission is $28.9 \%$. Results of univariable regression model showed that age, gender, hypertension, diabetes, chronic kidney disease, admission white cell counts, lymphocyte counts, D-Dimer, aspartate aminotransferase, alanine aminotransferase, insulin treatment, corticosteroid use, admission blood glucose, glucose coefficient of variation (CV), median blood glucose, median in-hospital glucose level, maximum blood glucose and minimum blood glucose were significantly associated with progression from non-critical cases to critical cases/ death $(\mathrm{p}<0.05)$ (online supplementary table S2). In the multivariable Cox model, admission blood glucose level was an independent risk factor for progression to critical cases/death (HR $=1.30,95 \%$ CI 1.03 to $1.63, \mathrm{p}=0.026)$ (table 2). For other glucose-associated indexes, multivariable Cox models showed that the median blood glucose $(\mathrm{HR}=1.09,95 \%$ CI 1.06 to 1.12$)$, glucose $\mathrm{CV}(\mathrm{HR}=2.40$, $95 \%$ CI 1.10 to $5.25, \mathrm{p}=0.028$ ), maximum blood glucose $(\mathrm{HR}=1.07,95 \%$ CI 1.04 to 1.09$)$ and minimum blood glucose (HR=1.07, 95\% CI 1.04 to 1.10$)$ were significant independent risk factors for progression to critical cases/ death (figure 2A). We found that higher median in-hospital glucose level was independently associated with the higher rate of progression to critical cases/death among non-critical cases (HR=2.25, 95\% CI 1.78 to 2.84 , $\mathrm{p}<0.001)$. Sensitivity analysis were performed in patients without diabetes, and multivariable regression model showed that the independent risk factors were the same to the analysis in all cases. Details were shown in online supplementary figure S1A.

\section{Blood glucose level was an independent risk factor for in- hospital mortality in critical cases}

As for the 697 critical cases, the time of critical diagnosis was available in 556 patients who were enrolled into the univariable and multivariable analysis. One hundred and fifty-one out of 556 patients died. The 30-day in-hospital mortality for critical cases is $30.9 \%$. Univariable analysis revealed that age, gender, smoking history, admission white cell counts, lymphocyte counts, D-Dimer, 
Table 1 Demographics and baseline characteristics of non-critical patients with COVID-19 at admission and critical patients with COVID-19 at the time of critical diagnosis

\begin{tabular}{|c|c|c|}
\hline Variables & Non-critical patients $(n=1690)$ & Critical patients $^{*}(n=697)$ \\
\hline Gender (female), n (\%) & $865(51.2)$ & $322(46.2)$ \\
\hline Age (years), median (IQR) & $61.0(50.0-69.0)$ & $65.0(54.0-73.0)$ \\
\hline Diabetes, n (\%) & $206(12.2)$ & $126(18.1)$ \\
\hline Hypertension, n (\%) & $418(24.7)$ & $244(35.0)$ \\
\hline Chronic obstructive pulmonary disease, $\mathrm{n}(\%)$ & $51(3.0)$ & $34(4.9)$ \\
\hline Cancert, n (\%) & $22(1.3)$ & $12(1.7)$ \\
\hline Chronic kidney disease, $\mathrm{n}(\%)$ & $33(2.0)$ & $25(3.6)$ \\
\hline Smoking history, $\mathrm{n}(\%)$ & $230(13.6)$ & $137(19.7)$ \\
\hline Admission glucose (mmol/L), $\mathrm{n}(\%)$ & $5.9(5.1$ to 7.5$)$ & - \\
\hline Normal $(<6.1)$ & $916(54.2)$ & - \\
\hline Hyperglycemia $(\geq 6.1)$ & $774(45.8)$ & - \\
\hline Initial glucose $(\mathrm{mmol} / \mathrm{L}) \neq, \mathrm{n}(\%)$ & - & $6.9(5.7$ to 8.7$)$ \\
\hline Normal $(<6.1)$ & - & $170(33.7)$ \\
\hline Hyperglycemia $(\geq 6.1)$ & - & $334(66.3)$ \\
\hline Occurrence of hypoglycemia, $\mathrm{n}(\%)$ & $79(4.7)$ & $26(5.2)$ \\
\hline \multicolumn{3}{|l|}{ Median blood glucose, $\mathrm{n}(\%)$} \\
\hline$<6.1$ & $973(57.6)$ & $166(32.9)$ \\
\hline$\geq 6.1$ & $717(42.4)$ & $338(67.1)$ \\
\hline Median glucose (mmol/L), median (IQR) & $5.8(5.1-7.1)$ & $6.9(5.7-8.5)$ \\
\hline Glucose coefficient of variation, median (IQR) & $0.2(0.1-0.3)$ & $0.2(0.1-0.3)$ \\
\hline Maximum glucose (mmol/L), median (IQR) & $6.7(5.4-8.8)$ & $8.4(6.6-11.4)$ \\
\hline Minimum glucose (mmol/L), median (IQR) & $5.2(4.6-6.2)$ & $5.7(4.7-7.2)$ \\
\hline Admission white blood cell counts $\left(10^{9} / \mathrm{L}\right)$, median (IQR) & $5.3(4.1-6.9)$ & $5.7(4.3-8.1)$ \\
\hline Admission lymphocyte counts (109/L), median (IQR) & $0.9(0.7-1.4)$ & $0.8(0.6-1.2)$ \\
\hline Admission D-Dimer (mg/L), median (IQR) & $0.4(0.2-0.8)$ & $0.5(0.3-1.1)$ \\
\hline Admission AST (U/L), median (IQR) & $25.3(18.0-38.0)$ & $29.6(19.6-46.7)$ \\
\hline Admission ALT (U/L), median (IQR) & $23.0(15.6-36.0)$ & $24.7(17.0-39.6)$ \\
\hline Admission creatinine $(\mu \mathrm{mol} / \mathrm{L})$, median $(\mathrm{IQR})$ & $69.0(57.0-84.0)$ & $72.5(59.9-95.0)$ \\
\hline Corticosteroid use, n (\%) & $653(38.6)$ & $373(53.5)$ \\
\hline Insulin use, n (\%) & $198(11.7)$ & $135(19.4)$ \\
\hline Oxygen therapy, n (\%) & $1601(94.7)$ & $682(97.8)$ \\
\hline Mechanical ventilation, $\mathrm{n}(\%)$ & $328(19.4)$ & $564(80.9)$ \\
\hline Admission to ICU, n (\%) & $132(7.8)$ & $342(49.1)$ \\
\hline Shock, n (\%) & $15(0.9)$ & $29(4.2)$ \\
\hline Median length of in-hospital stay (days), median (IQR) & $13.3(7.7-20.9)$ & $15.8(8.4-23.1)$ \\
\hline Death, n (\%) & $125(7.4)$ & $157(22.5)$ \\
\hline
\end{tabular}

*All the glucose-associated indices in critical patients with COVID-19 were analyzed based on the blood glucose concentration after diagnosis of critical case.

tIncluded in this category is any type of cancer.

$\ddagger$ The initial glucose of critical cases was the first blood glucose concentration after diagnosis of critical case.

ALT, alanine aminotransferase; AST, aspartate aminotransferase; ICU, intensive care unit.

creatinine, insulin treatment, corticosteroid use, initial blood glucose level of critical diagnosis, median blood glucose, median glucose level after critical diagnosis, maximum blood glucose and minimum blood glucose were significantly associated with in-hospital mortality $(\mathrm{p}<0.05) \quad$ (online supplementary table S3). In multivariable analysis, initial blood glucose level of critical diagnosis was significantly associated with in-hospital mortality (HR=1.84, 95\% CI 1.14 to 2.98, $\mathrm{p}=0.013$ ) (table 2). Regarding the other blood glucose-associated 
Table 2 Association of blood glucose group with progression to critical cases/death from non-critical cases at admission in patients with COVID-19 and with in-hospital mortality in critical cases of COVID-19 in multivariable Cox regression model ${ }^{*}$

\begin{tabular}{|c|c|c|c|c|}
\hline \multirow[b]{2}{*}{ Variables } & \multicolumn{2}{|l|}{ Non-critical patients } & \multicolumn{2}{|l|}{ Critical patients $\dagger$} \\
\hline & Adjusted HR (95\% Cl) & $P$ value & Adjusted HR (95\% Cl) & $P$ value \\
\hline \multicolumn{5}{|l|}{ Admission glucose group } \\
\hline Normal $(<6.1)$ & 1.00 & & 1.00 & \\
\hline Hyperglycemia ( $\geq 6.1$ ) & 1.30 (1.03 to 1.63$)$ & 0.026 & 1.84 (1.14 to 2.98$)$ & 0.013 \\
\hline Gender (female) & 0.86 (0.70 to 1.06$)$ & 0.157 & 0.43 (0.28 to 0.66$)$ & $<0.001$ \\
\hline Age (year) & 1.02 (1.01 to 1.02$)$ & $<0.001$ & 1.03 (1.02 to 1.05$)$ & $<0.001$ \\
\hline Diabetes & $1.03(0.72$ to 1.48$)$ & 0.851 & 1.26 (0.72 to 2.22$)$ & 0.415 \\
\hline Hypertension & 1.21 (0.95 to 1.53$)$ & 0.125 & 0.80 (0.52 to 1.22$)$ & 0.293 \\
\hline Smoking history & $1.17(0.88$ to 1.55$)$ & 0.275 & 1.36 (0.89 to 2.08$)$ & 0.162 \\
\hline Insulin treatment & 1.09 (0.78 to 1.51$)$ & 0.616 & 0.94 (0.57 to 1.54$)$ & 0.807 \\
\hline Systemic glucocorticoids & 1.77 (1.40 to 2.22$)$ & $<0.001$ & 4.47 (2.41 to 8.30$)$ & $<0.001$ \\
\hline Chronic kidney disease & $2.24(1.14$ to 4.41$)$ & 0.019 & $0.50(0.14$ to 1.78$)$ & 0.284 \\
\hline Chronic obstructive pulmonary disease & 0.96 (0.55 to 1.66$)$ & 0.885 & $0.58(0.26$ to 1.28$)$ & 0.179 \\
\hline Cancer & $0.76(0.33$ to 1.75$)$ & 0.519 & 0.66 (0.18 to 2.43$)$ & 0.534 \\
\hline Admission white cell counts & $1.10(1.07$ to 1.13$)$ & $<0.001$ & $1.10(1.06$ to 1.14$)$ & $<0.001$ \\
\hline Admission lymphocyte counts & $0.55(0.42$ to 0.71$)$ & $<0.001$ & $0.63(0.37$ to 1.07$)$ & 0.090 \\
\hline Admission D-Dimer & $1.06(1.03$ to 1.09$)$ & $<0.001$ & $1.02(0.99$ to 1.04$)$ & 0.267 \\
\hline Admission AST & $1.00(1.00$ to 1.00$)$ & 0.157 & $1.00(1.00$ to 1.01$)$ & 0.106 \\
\hline Admission ALT & $1.00(0.99$ to 1.00$)$ & 0.684 & 0.99 (0.98 to 1.00$)$ & 0.185 \\
\hline Admission creatinine & 1.00 (1.00 to 1.00$)$ & 0.102 & $1.00(1.00$ to 1.00$)$ & $<0.001$ \\
\hline
\end{tabular}

*Multivariable Cox analysis was adjusted for all available factors in baseline table.

†The glucose-associated indices of critical cases were analyzed based on the blood glucose concentration after diagnosis of critical case.

ALT, alanine aminotransferase; AST, aspartate aminotransferase.

indexes, multivariable analysis showed that median blood glucose (HR=1.09, 95\% CI 1.04 to 1.14$)$ and minimum blood glucose (HR=1.14, 95\% CI 1.09 to 1.19$)$ were significant independent risk factors for in-hospital mortality of critical cases (figure 2B). Higher median glucose level after critical diagnosis was independently associated with higher rate of in-hospital mortality in critical cases $(\mathrm{HR}=2.39,95 \%$ CI 1.41 to $4.07, \mathrm{p}=0.001)$. Sensitivity analysis in critical patients without diabetes were also performed. Multivariable regression model showed that the initial blood glucose of critical diagnosis, blood glucose control, median blood glucose and minimum blood glucose were independent risk factors for the in-hospital mortality of critical cases without diabetes. Details were shown in online supplementary figure S1B.

\section{DISCUSSION}

Based on the data from 2041 consecutive patients with COVID-19, we found that admission blood glucose level was an independent risk factor for predicting the progression to critical cases/death from non-critical cases, and initial blood glucose level of critical diagnosis was an independent risk factor for in-hospital mortality in critical cases. In addition, patients with higher median glucose level during hospital stay or after critical diagnosis had significantly poorer clinical outcomes. The above results were also confirmed in COVID-19 patients without a history of diabetes.

The current pandemic of COVID-19 caused a global health crisis. It has been reported that progression into critical cases could happen within 3-10 days in 10\%-20\% of cases. ${ }^{12}{ }^{13}$ Our data showed that critical cases consisted of $34.1 \%$ of the COVID-19 patient population, with a 30-day mortality of $30.7 \%$. Therefore, predicting the likelihood of progression to critical cases in non-critical cases as well as predicting in-hospital mortality in critical cases became particularly important to the stratified management of patients with COVID-19 in a circumstance of severe shortage of medical resources during pandemic. As a convenient and easy-to-detect index, blood glucose level can be obtained and monitored in all clinical settings. Our data showed that $47.2 \%$ of patients with COVID-19 had elevated blood glucose levels at admission, and blood glucose level was an independent risk factor for progression to critical cases/death in noncritical cases. Furthermore, analysis of critical cases found that initial blood glucose level of critical diagnosis was an independent factor for in-hospital mortality in critical cases, indicating that blood glucose levels may serve as an instant and simple parameter for risk stratification 




Figure 2 Forest plots of the association of multiple glucose indexes (A) with progression to critical cases/death among noncritical COVID-19 patients at admission and (B) with in-hospital mortality among critical patients with COVID-19 in multivariable Cox regression analysis.

and hierarchical management of COVID-19 in all clinical settings.

Interestingly, while $47.2 \%$ of the patients had admission hyperglycemia, only $13.4 \%$ of the patients had diabetes. Diabetes has been previously reported to affect the outcomes of COVID-19 cases. ${ }^{3}$ However, the predictive value of blood glucose level in patients without diabetes are more concerning in clinical practice. Elevation of the blood glucose level may represent relative hyperglycemia. Infection might trigger an inflammatory storm, which leads to insulin resistance. Infection could also induce stress and sympathetic stimulation. The SARS-CoV-2 virus might also directly attack the pancreas. All these factors may render infected COVID-19 patients more prone to hyperglycemia. ${ }^{14} 15$ Therefore, we performed analysis in patients without diabetes. Results are consistent with the overall findings. These results further illustrated that blood glucose could reliably predict the risk of hospitalized patients with COVID-19.

Our study showed that although the rate of hypoglycemia was higher $(6.7 \%)$ in patients with normal median blood glucose level as compared with that in patients with high median blood glucose level $(>6.1 \mathrm{mmol} / \mathrm{L})$
$(2.0 \%)$, the overall mortality was significantly reduced. This finding was different from prior study that median blood glucose level $\leq 6.1 \mathrm{mmol} / \mathrm{L}$ might increase the risk of both hypoglycemia and mortality in patients with diabetes. ${ }^{16}$ A possible explanation is that previous studies targeted patients with diabetes, and hypoglycemia is more likely to induce cardiovascular events, leading to increased mortality. However, for patients with COVID19, the prevalence of cardiovascular comorbidities was actually lower than that of patients with diabetes. Thus, a slight increase in the risk of hypoglycemia did not offset the survival benefits of reducing blood glucose level. This result underlies the importance of close monitoring and control of blood glucose level during the treatment of COVID-19. Of note, in light of the potential risk of hypoglycemia, glucose control should be personalized, especially in patients with comorbidities such as cardiovascular diseases.

There are several limitations in the current study. First, the study was conducted during a large-scale infectious disease outbreak setting when the healthcare system was overwhelmed by large number of patients seeking medical care. It was a retrospective cohort study in nature, since 
conducting a large randomized trial was too challenging. Second, detailed data on non-insulin diabetes medications and fingerstick blood glucoses done in-between were lacking in this study. Third, the current study only included hospitalized patients, which were more severely ill cases. Hence, the generalizability of these findings to non-hospitalized patients with milder disease needs to be further verified.

In summary, our study found that blood glucose level was an independent risk factor to predict the progression to critical cases/death in non-critical cases and in-hospital mortality in critical cases, whereas patients with higher median glucose level during hospital stay or after critical diagnosis had significantly poorer clinical outcomes. Our results provided a simple and practical way to risk stratify COVID-19 inpatients for hierarchical management, particularly where medical resources are in severe shortage during the pandemic.

\section{Author affiliations}

${ }^{1}$ Department of Critical Care Medicine, The First Affiliated Hospital of Sun Yat-sen University, Guangzhou, Guangdong, China

${ }^{2}$ Division of Pulmonary and Critical Care Medicine, The First Affiliated Hospital of Sun Yat-sen University, Guangzhou, Guangdong, China

${ }^{3}$ Department of Critical Care Medicine, The Affiliated Hospital of Jianghan University (No. Six Hospital of Wuhan), Wuhan, China

${ }^{4}$ Department of Critical Care Medicine, Wuhan Hankou Hospital, Wuhan, China

${ }^{5}$ Department of Gastrointestinal Surgery, Wuhan Hankou Hospital, Wuhan, China

${ }^{6}$ Science and Education Section, Wuhan Hankou Hospital, Wuhan, China

${ }^{7}$ Department of Gastrointestinal Surgery, The Affiliated Hospital of Jianghan

University (No. Six Hospital of Wuhan), Wuhan, China

${ }^{8}$ Clinical Trials Unit, The First Affiliated Hospital of Sun Yat-sen University,

Guangzhou, Guangdong, China

${ }^{9}$ Department of Endocrinology, The First Affiliated Hospital of Sun Yat-sen University, Guangzhou, Guangdong, China

${ }^{10}$ Department of Medical Ultrasonics, The First Affiliated Hospital of Sun Yat-sen University, Guangzhou, Guangdong, China

${ }^{11}$ Department of Liver Surgery, The First Affiliated Hospital of Sun Yat-sen University, Guangzhou, Guangdong, China

${ }^{12}$ Department of Cardiology, The First Affiliated Hospital of Sun Yat-sen University, Guangzhou, Guangdong, China

${ }^{13}$ Department of Medicine and Therapeutics, The Chinese University of Hong Kong, Hong Kong, China

${ }^{14}$ Precision Medicine Institute, The First Affiliated Hospital of Sun Yat-sen University, Guangzhou, Guangdong, China

Acknowledgements We would like to thank all the hospital staff members for their efforts in working in the frontline.

Contributors HaiX supervised the study. HaiX, JW, JH, GZ, QW, QL and YH designed the study. XG, CW and JS helped to organize the study. JW, JH, GZ, QW $\mathrm{QL}, \mathrm{YH}, \mathrm{YaL}, \mathrm{YY}, \mathrm{XS}$ and $\mathrm{HY}$ collected the data. QZ did the data analysis. JW, SP, YiL, HaiX, XL and DY wrote the draft report. JS performed critical revision on the manuscript. All authors contributed to the analysis and interpretation of data. All authors approved the final version before submission.

Funding The authors have not declared a specific grant for this research from any funding agency in the public, commercial or not-for-profit sectors.

Competing interests None declared.

Patient consent for publication Not required.

Ethics approval The study is approved by the ethics committees of Wuhan Hankou Hospital, No. Six Hospital of Wuhan and the First Affiliated Hospital of Sun Yat-sen University.
Provenance and peer review Not commissioned; externally peer reviewed.

Data availability statement Data are available on reasonable request. Data used during the study are available from the corresponding author by request.

Open access This is an open access article distributed in accordance with the Creative Commons Attribution Non Commercial (CC BY-NC 4.0) license, which permits others to distribute, remix, adapt, build upon this work noncommercially, and license their derivative works on different terms, provided the original work is properly cited, appropriate credit is given, any changes made indicated, and the use is non-commercial. See: http://creativecommons.org/ licenses/by-nc/4.0/.

ORCID iDs

Yihao Liu http://orcid.org/0000-0003-3270-9797

Yanbing Li http://orcid.org/0000-0001-7242-760X

Haipeng Xiao http://orcid.org/0000-0002-4188-336X

\section{REFERENCES}

1 World Health Organization. Coronavirus disease (COVID-19) pandemic [article online]. Available: https://www.who.int [Accessed 11 Mar 2020].

2 World Health Organization. Coronavirus disease (COVID-2019) situation reports [article online]. Available: https://www.who.int/ emergencies/diseases/novel-coronavirus-2019/situation-reports [Accessed 9 Apr 2020].

3 Guan W, Liang W, Zhao Y, et al. Comorbidity and its impact on 1,590 patients with COVID-19 in China: a nationwide analysis. medRxiv 2020.

4 Epidemiology Working Group for NCIP Epidemic Response, Chinese Center for Disease Control and Prevention. [The epidemiological characteristics of an outbreak of 2019 novel coronavirus diseases (COVID-19) in China]. Zhonghua Liu Xing Bing Xue Za Zhi 2020;41:145-51.

5 Truog RD, Mitchell C, Daley GQ. The Toughest Triage - Allocating Ventilators in a Pandemic. N Engl J Med 2020;382:1973-5.

6 Lee TF, Drake SM, Roberts GW, et al. Relative hyperglycemia is an independent determinant of in-hospital mortality in patients with critical illness. Crit Care Med 2020;48:e115-22.

7 Kalfon P, Le Manach Y, Ichai C, et al. Severe and multiple hypoglycemic episodes are associated with increased risk of death in ICU patients. Crit Care 2015;19:153.

8 National Health Commission of the People's Republic of China. The 7th trial version of diagnosis and treatment scheme for pneumonitis caused by COVID-19 infection (in Chinese), 2020. Available: http:// www.nhc.gov.cn/yzygj/s7653p/202003/46c9294a7dfe4cef80dc 7f5912eb1989/files/ce3e6945832a438eaae415350a8ce964.pdf [Accessed 4 Mar 2020].

9 Yamada T, Shojima N, Noma H, et al. Glycemic control, mortality, and hypoglycemia in critically ill patients: a systematic review and network meta-analysis of randomized controlled trials. Intensive Care Med 2017:43:1-15.

10 Vistisen D, Witte DR, Brunner EJ, et al. Risk of cardiovascular disease and death in individuals with prediabetes defined by different criteria: the Whitehall II study. Diabetes Care 2018;41:899-906.

11 Kwan TN, Zwakman-Hessels L, Marhoon N, et al. Relative hypoglycemia in diabetic patients with critical illness. Crit Care Med 2020;48:e233-40.

12 Chen N, Zhou M, Dong X, et al. Epidemiological and clinical characteristics of 99 cases of 2019 novel coronavirus pneumonia in Wuhan, China: a descriptive study. Lancet 2020;395:507-13.

13 Wang D, Hu B, Hu C, et al. Clinical characteristics of 138 hospitalized patients with 2019 novel coronavirus-infected pneumonia in Wuhan, China. JAMA 2020;323:1061-9.

14 Marik PE, Raghavan M. Stress-hyperglycemia, insulin and immunomodulation in sepsis. Intensive Care Med 2004;30:748-56.

15 Šestan M, Marinović S, Kavazović I, et al. Virus-Induced interferon- $\gamma$ causes insulin resistance in skeletal muscle and derails glycemic control in obesity. Immunity 2018;49:164-77.

16 Yamada T, Shojima N, Noma H, et al. Glycemic control, mortality, and hypoglycemia in critically ill patients: a systematic review and network meta-analysis of randomized controlled trials. Intensive Care Med 2017:43): :1-15 